\title{
Re-irradiation is now a real option - but how do we take it forward?
}

Rebecca Muirhead ${ }^{1}$, Bleddyn Jones ${ }^{2}$

${ }^{1}$ Oxford Cancer Centre, Oxford University Hospitals NHS Foundation Trust, Oxford, UK

${ }^{2}$ CRUK MRC Oxford Institute for Radiation Oncology, University of Oxford, Oxford, UK

Corresponding author:

Rebecca Muirhead

Level 2 Cancer Administration offices

Oxford Cancer Centre

Churchill Hospital

Old Road

Oxford

OX3 7LE

Telephone: 07989445591

Fax: 01865235981

Type of Article:

Editorial

Conflicts of Interest:

The authors have no conflicts of interest to report.

Word Count:

946

Number of tables and charts:

0 
Editorial

Re-irradiation is Now a Real Option - But How Do We Take it Forward?

R. Muirhead *, B. Jones $†$

* Oxford Cancer Centre, Oxford University Hospitals NHS Foundation Trust, Oxford, UK

${ }^{\dagger}$ CRUK MRC Oxford Institute for Radiation Oncology, University of Oxford, Oxford, UK

Received and accepted 22 November 2017

Author for correspondence: R. Muirhead, Level 2 Cancer Administration Offices, Oxford Cancer Centre, Churchill Hospital, Old Road, Oxford OX3 7LE, UK. Tel: +447989445591; Fax: +44-1865-235981.

E-mail address: rebeccamuirhead@hotmail.com (R. Muirhead).

The decision to offer radiotherapy may appear to be a routine procedure to many practitioners, but it should never be taken lightly: good judgement, with inclusion of scientific and clinical evidence, the views of the patient and other disciplines involved in the management of cancer. Decisions to retreat with overlapping fields, or with full inclusion of the previously treated area, require an even greater depth of critical assessment, as there may be greater serious tissue complications and long-term tumour control consequences if certain conditions are not met.

To date, with conformal treatments and perhaps due to a lack of robust late toxicity data, there has been a reluctance to offer re-irradiation unless in a palliative setting for symptom control. But even then, previous and planned dose and fractionation and inclusion of important structures such as the spinal cord, brain, heart, kidneys or large vessels can impinge on the decision in subtle ways. The prognosis of the patient has a considerable influence; and it is a balance between what degree and length of palliation can be obtained, against a realistic assessment of the time required for the development of late adverse effects. In our introductory overview in this special issue, Dörr and Gabryś [1] discuss the common organs at risk, detailing knowledge to date from animal and human studies.

Radiotherapy has changed significantly in the last few decades, with improved delivery techniques and planning algorithms, protons and heavier ions becoming more readily available and other supplementary therapies, such as hyperthermia, under investigation, all potentially enhancing re-irradiation tumour control while sparing organs at risk. As such, there is a renewed interest in re-irradiation in multiple tumour sites. Hyperthermia is discussed in some detail by Kaidar-Person et al. [2], specifically in the context of breast cancer re-irradiation. The delivery of re-irradiation specifically using SABR is discussed, in the re-irradiation of vertebral body metastasis by Myrehaug et al. [3] and a series of patients irradiated for biochemical relapse of prostate cancer by Loi et al. [4].

Nearly two decades ago, the British Journal of Radiology published a commentary following a re-irradiation meeting encouraging clinical oncologists to think about safe reirradiation [5]. It discussed the possibility of management guidelines for re-irradiation. However, there are some common themes throughout the different tumour types, a major one being that guidance is a major challenge in such a complex treatment delivery, probably 
due to the heterogeneity of situations in which it would occur and the lack of robust data. Some tumour types may feel that site-specific guidance is appropriate, but this would have to be developed with a large national multidisciplinary team. All clinicians, irrelevant of treatment site, should undertake a thorough history and examination to identify residual late toxicity from the initial radiation course. Adequate informed consent is vital, with thorough discussion of alternative treatments, covering the aims, risks and benefits. None of the tumour types has gold standard evidence or robust late toxicity data. Therefore, clinicians must be cautious in their description of potential risks. If trials are not immediately available, in the interim there is a need for large series with homogeneous delivery, good quality assurance and prospective data collection to enable adequate evaluation of reirradiation. The UK has an excellent history of collaborating to deliver a homogeneous quality of radiotherapy through national guidance, education, mentoring and collection of national data using thorough audit. This would need to be site-specific, including applications for funding and buy in from major centres. It should encourage recording of a minimal data set inclusive of tumour stage, site, treatment volumes, dose-fractionation schedule, time to recurrence, as well as similar recording of the same parameters for retreatments, inclusive of relevant histories such as surgical interventions and chemotherapies given. These data could be used to construct models that would discriminate between retreatments with favourable and unfavourable outcomes, as well as furnish further information on which to base the choice of dose fractionation, using a BED or EQD2 approach - all informing future research and development.

The different overviews also highlight the variations in aims and risks of reirradiation in different tumour types. For example, in lung and gynaecological cancers, radical re-irradiation is delivered when alternative treatment strategies are not possible, as described by Rulach et al. [6] and Sadozye [7], respectively. Rectal re-irradiation is used to either improve symptom control or is given as part of radical multidisciplinary treatment in conjunction with surgery, as discussed by Owens and Muirhead [8]. By contrast, Ho and Jena [9] describe the use of re-irradiation only with palliative intent with the aim of neurological symptom relief and to reduce steroid dependency. It is therefore impossible to have guidance on re-irradiation in general and site-specific guidance is more appropriate.

Further avenues of investigation could include assessment of the tolerance of different organs at risk allowing for multiple factors, and the importance of time-dependent increments in the available tissue tolerance, at least in some tissues such as the central nervous system. For example, a downloadable graphical user interface has been developed in Oxford for changes in spinal cord retreatment tolerance with increasing time between initial and retreatments. This includes all the available animal data and applies to all doses and potential time intervals between treatment courses [10] and has the potential to be applied in other central nervous system retreatments. These developments can act as a stimulant to encourage similar development in other anatomical sites and organs at risk.

We hope the overviews within this special issue act not only as a tool to inform clinicians considering re-irradiation, but also encourage relevant groups to consider national guidelines and data collection and stimulate discussion regarding potential clinical trials in the more commonly retreated anatomical sites.

\section{References}


[1] Dörr W, Gabryś D. The principles and practice of re-irradiation in clinical oncology: an overview. Clin Oncol 2017;29:000-000.

[2] Kaidar-Person O, Oldenborg S, Poortmans P. Re-irradiation and hyperthermia in breast cancer. Clin Oncol 2017;29:000-000.

[3] Myrehaug S, Soliman H, Tseng C, Heyn C, Sahgal A. Re-irradiation of vertebral body metastases: treatment in the radiosurgery era. Clin Oncol 2017;29:000-000.

[4] Loi M, Di Cataldo V, Simontacchi G, Detti B, Bonomo P, Masi L, Desideri I et al. Robotic stereotactic retreatment for biochemical control in previously irradiated patients affected by recurrent prostate cancer. Clin Oncol 2017;29:000-000.

[5] Jones B, Blake PR. Commentary: Re-treatment of cancer after radical radiotherapy. BJR 1999;72:1037-9.

[6] Rulach R, Hanna GG, Franks K, McAleese J, Harrow S. Re-irradiation for locally recurrent lung cancer: evidence, risks and benefits. Clin Oncol 2017;29:000-000.

[7] Sadozye AH. Re-irradiation in gynaecological malignancies: a review. Clin Oncol 2017;29:000-000.

[8] Owens R, Muirhead R. External beam re-irradiation in rectal cancer. Clin Oncol 2017;29:000-000.

[9] Ho ALK, Jena R. Re-irradiation in the brain: primary gliomas. Clin Oncol 2017;29:000-000. [10] Woolley et al. Int J Radiat Biol (in press). 